Toribio-Guzmán, J. M., García-Holgado, A., Soto Pérez, F., García-Peñalvo, F. J., \& Franco Martín, M. Á. (2016). Heuristic evaluation of SocialNet, the private social network for psychiatric patients and their relatives. In F. J. García-Peñalvo (Ed.), Proceedings of the Fourth International Conference on Technological Ecosystems for Enhancing Multiculturality (TEEM'16) (Salamanca, Spain, November 2-4, 2016) (pp. 525-532). New York, NY, USA: ACM. doi:10.1145/3012430.3012568

\title{
Heuristic evaluation of SocialNet, the private social network for psychiatric patients and their relatives
}

\author{
José Miguel Toribio-Guzmán \\ Iberian Institute for Research in \\ Psychosciences, INTRAS Foundation \\ Ctra. de la Hiniesta 137. 49024 Zamora, \\ Spain \\ +34980516427 \\ investigacion4@intras.es
}

\author{
Alicia García-Holgado \\ GRIAL Research Group, \\ Computer Sciences Department, \\ University of Salamanca \\ Paseo de Canalejas 169, 37008 \\ Salamanca, Spain \\ +34923294500 ext. 3433 \\ aliciagh@usal.es
}

\author{
Felipe Soto Pérez \\ Iberian Institute for Research in \\ Psychosciences, INTRAS Foundation \\ Ctra. de la Hiniesta 137. 49024 Zamora, \\ Spain \\ +34980516427 \\ psicoeac@intras.es
}
Francisco J. García-Peñalvo GRIAL Research Group, Computer Sciences Department, University of Salamanca
Paseo de Canalejas 169, 37008
Salamanca, Spain
+34 923294500 ext. 3433
fgarcia@usal.es

\author{
Manuel A. Franco Martín \\ Department of Psychiatry of Mental Health, Zamora Hospital/ \\ Department of Personality, Assessment and Psychological \\ Treatment (University of Salamanca) Hernán Cortés, 40. 49021 \\ Zamora, Spain \\ +34980520200 \\ mfm@intras.es
}

\begin{abstract}
SocialNet, is a private social network for monitoring daily progress of patients by their relatives. The goal of this paper is evaluate the usability of SocialNet in order to improve it and provide users an effective, efficient interaction and satisfactory experience. This work presents the first phase where it was used a double heuristic evaluation with one expert evaluator. This technique has provided valuable data about the usability of SocialNet especially related to consistency, design and privacy. The tool has been redesigned in order to resolve the identified problems as part of an iterative process. The use of heuristic evaluation will be complemented by a user test to be carried out in a second phase of usability study, which will not be addressed in this paper.
\end{abstract}

\section{CSS concepts}

• Networks Online social networks • Human-centered computing Heuristic evaluations • Applied computing Health care information systems

Keywords

Usability; Heuristic Evaluation; Social Network; Information System; Patient Care System; Web.

\section{INTRODUCTION}

The use of new Information and Communication Technologies (ICT) is widespread, $46 \%$ of the world population are Internet users and $51 \%$ use mobile devices [15]. All these tools have provided a wide range of possibilities in all areas of life. In particular, the medical area has used ICT tools not only to support the daily activities in hospitals and other health centers, but also to improve treatments. For example, in the case of psychiatric services, Internet has allowed the use of alternative therapies that otherwise could not receive them.

In this technological context, the Psychiatric Department of the Zamora Hospital (Spain) and INTRAS, a private non-profit foundation, have made a proposal for improving the treatment of patients with mental disorders and addictions.

Psychiatric treatment differs very much from other medical specialties treatments, because most of them are done outside of the hospital environment, either in Day Centers, supervised apartments or Community Rehabilitation Centers addressed to promote their rehabilitation and social integration. INTRAS Foundation collaborates with the Hospital of Zamora for providing a comprehensive and continuous care depending on the type of disorder or mental addiction, taking into account the features and follow-up of the patient.

Every patient under psychiatric treatment has a unique personal situation due to both their social context and the heterogeneity of mental illness. Although there are patients without relatives or people nearby, most of them have people interested in their follow-up and wellbeing further than hospitalization. These links between relatives and patient are positive for prognosis of patient. Because of this, it is necessary to promote tools and methodologies for improving the communication between families or friends and users and professionals. In general terms, there are formal mechanisms to communicate medical information to authorized persons of the patient, but this information only focuses on the medical treatment or contingences happened, but not on the evolution of the patient outside the health center or positive achievements. On the other hand, there are many relatives living far to the patient house of hospital and because of this the links and communications among them are limited. If it is taken into account that the active inclusion of family members in the life of the patient has 
a positive impact in their process of improvement [9]. It will be necessary to develop tools for improving the communications with far family and for giving information about the achievements and positive activities of the patient without having a negative impact in their daily work.

In the last years have arisen virtual communities in which patients, families and health professionals share knowledge about a disease, a health problem or medical condition specific [17]. However, these tools do not provide an environment where people who are involved in the follow-up of the patient to be able to share information securely with people close to the patient and the family.

SocialNet is an online tool that provides a private social network for each patient, their families and any person authorized by him. Caregivers or others involved in the treatment and follow-up of the patient can share information about activities, meetings, achievements, etc., in a private technological environment.

The idea of this innovative tool started from the combination of the strengths of two different contexts. First of all, the educational context represented by the University of Salamanca through the GRIAL research group [5; 8], which provides knowledge about the development of information systems focusing on learning and knowledge management [2-4; 6]. Secondly, the medical context represented by the Hospital of Zamora and the Foundation INTRAS, for identifying unmet needs and provide a good environment to carry out the study of usability.

The goal of this paper is to evaluate the usability of SocialNet in order to make it a usable product and users may have an effective, efficient interaction and satisfactory experience. SocialNet is currently a functional prototype available at http://psiquiatria.grial.eu. This prototype has been used to carry out the study of usability.

The paper has been divided into four parts. The first part gives a description of the functional prototype of SocialNet. The second part describes the features of the usability evaluation. Third part is focused on the heuristic evaluation. Finally, the last part provides the main conclusions of this research.

\section{THE PRIVATE SOCIAL NETWORK}

SocialNet is based on Drupal (version 7.x) (http://drupal.org), an Open Source Content Management System (CMS). Although there are other CMS with similar characteristics, Drupal is the most advanced technically [16], with a powerful framework development, in addition to being the most advisable for the development of sites based on groups, communities or, in the case of SocialNet, social environments [22]. On the other hand, Drupal has a very active community of developers that develop different modules to extend the functionality of the kernel.

Private social network is composed of a set of private and safe areas for each patient. These areas are called walls (Figure 1), following the same metaphor used in other social networks such as Facebook or LinkedIn. The main difference between SocialNet and other existing social networks is that the patient is not only a user, but is the center of the interaction. In addition, each wall is a single social network, family members perceive the tool as a space for them, they cannot know if there are other walls or other users at SocialNet; This is part of the privacy measures.

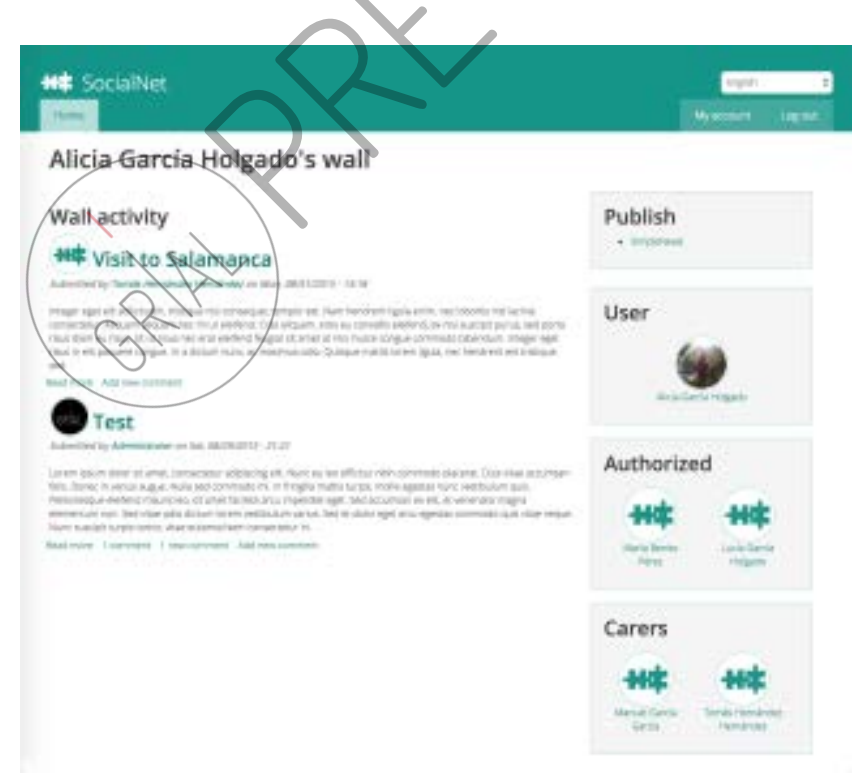

Figure 1. Example of a patient's wall

Security of Drupal as platform depends on modules that extend its functionality and configuration of the system itself [14]. In the case of SocialNet, several security measures have been used in order to ensure the privacy of the information stored in the walls of the patients. Besides it is necessary to highlight the management of users through a hierarchy of roles, combined with a careful definition of the permissions associated with each role.

There are two types of users: anonymous, only have access to the login page; and authorized, they can be patients, family members, caregivers, caregiver managers, general caregivers or administrators. The roles take into account a hierarchy, so that the passive user is the one with fewer permissions and the user administrator has all permissions. Patients and family members can have a role further, active or 
passive, in order to control your interaction within the wall. In the case of severe patients are usually passive users, and cannot publish or comment, but can read the publications or comments made by caregivers or other family members. By the other hand, family members usually are active users, can share images, videos and text, enriched with a set of metadata, in the wall of the patient, as well as qualify or make comments on publications.

Active users and caregivers of the patient make most of the interaction in the wall. Other roles with more permissions, as caregiver managers and general managers, are focused on ensuring the appropriate use of the social environment. García-Peñalvo et al. [7] provides detailed information about tool operation.

\section{USABILITY EVALUATION}

Usability is one of the most prominent criteria that must be fulfilled by a software product, in particular by a web application. Because of this, the main requirement for SocialNet should be providing a usable interface.

This study is focused on the usability evaluation of SocialNet. It presents a first assessment phase which involves the application of a usability inspection method based on experts: the heuristic evaluation [20]. Usability inspections are applied commonly, especially in the early stages of the system development [11], because of its simplicity and low cost has gained popularity and is often employed in studies of usability [13].

While the more robust approach for the evaluation of usability has to involve users, these will be the protagonists of a second phase (user Test) needed to complete the study of usability of SocialNet, which will not be described in this paper to be ongoing.

Usability evaluation methods can be defined as "procedures comprised a series of well-defined activities to collect data related to the interaction between the end user and a software product, in order to determine the specific properties of a software for contributing to the achievement of specific objectives" [1]. In general, these methods are used during all phases of the software development process to ensure a product design wearable and can meet the high quality standards.

There are several classifications of usability evaluation methods taking into account the different techniques used. For instance, the proposal which establish the follow usability evaluation methods: methods of inspection: research methods and Test methods [10] (Table $1)$.

Table 1. Usability evaluation methods [10]

\begin{tabular}{|c|c|}
\hline Methods & Techniques \\
\hline Inspection & $\begin{array}{ll}\text { - } & \text { Cognitive Walkthrough } \\
\text { - } & \text { Peuristic Evaluation } \\
\text { - } & \text { Formal Usability Inspection } \\
\text { - } & \text { Feature Inspection } \\
\text { - } & \text { Standards Inspection } \\
\text { - } & \text { Pensistency Inspection } \\
& \text { Perspective based Inspection }\end{array}$ \\
\hline & $\begin{array}{ll}\text { - } & \text { Field Observation } \\
\text { - } & \text { Focus Group } \\
\text { - } & \text { Questionnaires } \\
\text { - } & \text { Interviews } \\
& \text { Logging Actual Use }\end{array}$ \\
\hline Test & $\begin{array}{ll}\text { - } & \text { Thinking Aloud Protocol } \\
\text { - } & \text { Question Asking Protocol } \\
\text { - } & \text { Performance Measurement } \\
\text { - } & \text { Coaching Method } \\
\text { - } & \text { Teaching Method } \\
\text { - } & \text { Co-Discovery Learning } \\
\text { - } & \text { Retrospective Testing } \\
\text { - } & \text { Remote Testing }\end{array}$ \\
\hline
\end{tabular}

Specially, usability inspection method is one of those methods and heuristic evaluation one of its most prominent techniques.

Heuristic evaluation is a method developed to test interfaces in a fast and economical way. It consists in finding usability problems in the design of the interface, which can have a negative effect on the ability of the user to interact with the system, so that these can be corrected as part of an iterative design process. One or more usability experts examine each element of the user interface and verify if the proposed design is in keeping with recognized usability principles called "heuristics" [12].

As stated above, among the advantages of this technique are its low cost, and its ease of use and also allows finding many usability problems. However, the most important disadvantage is to rely on the results of the assessment in excess of the experience subjective evaluators. 


\section{SOCIALNET HEURISTIC EVALUATION}

In order to carry out the heuristic evaluation of SocialNet have been used heuristic rules proposed by Nielsen [18]:

1. Visibility of system status.

2. Match between system and the real world.

3. Freedom and user control.

4. Consistency and standards.

5. Error prevention.

6. Better recognize to memorize.

7. Flexibility and efficiency of use.

8. Aesthetic and minimalist design.

9. Helps users recognize, diagnose, and recover from errors.

10. Help and documentation.

Besides, other heuristic has been added:

11. Privacy.

This heuristic proposed by Pierotti [21] has been considered necessary to include, because SocialNet is a private social network system for helping the user to protect personal information, both belonging to the user and his family.

According to Zazelenchuk [24], although the heuristic evaluation is an standard created over 20 years ago, many evaluators have found that the original list of Nielsen [19] does not always meet the specific needs for every stud, so in some cases it is required some re-interpretation of the original descriptions of Nielsen for every heuristic.

Regarding the number of experts to carry out a heuristic evaluation, Zazelenchuk [24] and Wilson [23] remarks that a heuristic evaluation consists of one or more experienced assessors. In the case of SocialNet, a single expert has made a double review.

First, the expert has used the website of SocialNet as a new user, without any previous experience. For each of the user profiles the expert has provided a brief description of the problems found and has selected the heuristic applied for each problem.

The results from the first study have provided relevant information to develop a new version of the social network in order to resolve the different identified problems.

Once released a new version of the functional prototype, the same expert has performed a second study. Although is the same evaluator, the experience with SocialNet is different because the evaluator has gained knowledge about the tool during the first study.

\subsection{Study 1}

For this first study, the expert evaluator uses SocialNet as a novel user receiving only a general explanation about the operation of the system and its features. Taking into account that it is a private social network that address to improve the communicative relationship between patients, families and caregivers, there are different application roles that form a hierarchical structure linked to different permissions to each one of them. So the task of the evaluator was to carry out the evaluation heuristic with each of the roles, with the exception of the role of the patient, since this is usually a passive user, who may not publish or comment, but can read the publications and comments made by caregivers or relatives. Besides, it was taken into account that patient and family member/authorized roles are similar, was chosen not to use the patient role.

\subsubsection{Results}

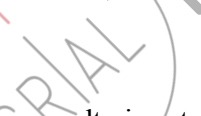

The first expert assessment has collected the results in a table that listed the number of problems clustered by heuristic and the number of problems clustered according to the user profile and heuristic. This table has helped to sort and summarize data quantitatively. First, the number of problems found for each heuristic was obtained. Figure 2 shows that the heuristic 4, 8 and 11 present greater number of problems, which correspond to consistency and standards (16 problems), aesthetic design and minimalist ( 7 problems) and privacy ( 9 problems). Other heuristics are not relevant because only one or no problem have been detected.

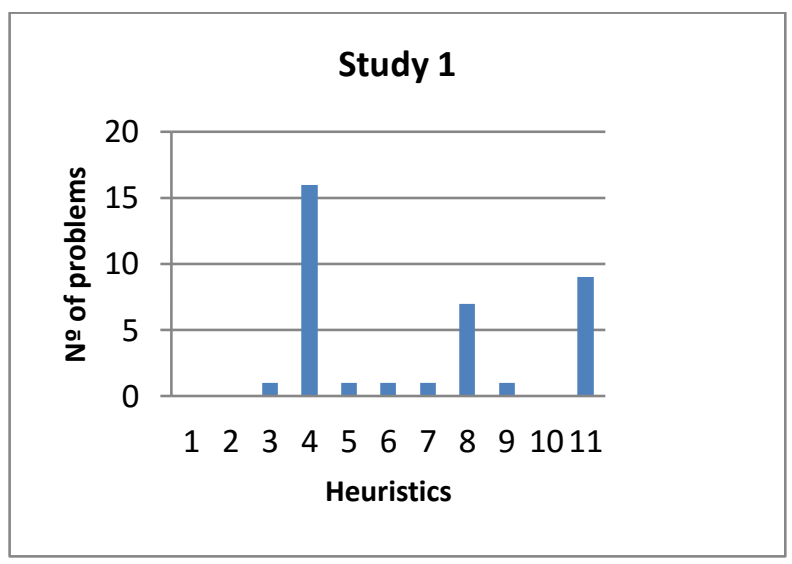

Figure 2. Number of problems found by heuristic in study 1 of SocialNet 
The evaluator established the three criticality levels for problems found: low level, minor usability problems which have low priority to fix them; medium level, usability problems which should be fixed with certain priority; high level, serious usability problem which should be solved with high priority. Each problem found is rated in one of these levels (Figure 3).

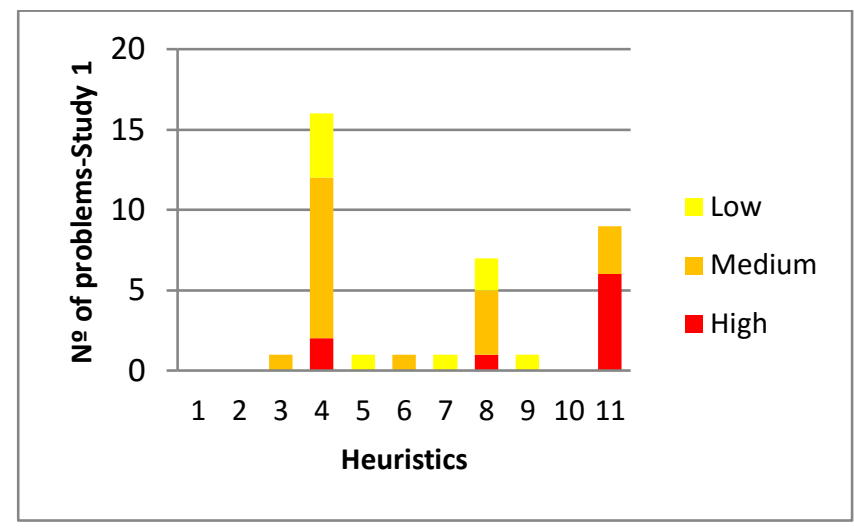

Figure 3. Criticality Level of the problems found in study 1

Overall, the criticality level of the problems found is medium-high, a large number of problems (30 of 39) were rated within these levels. Besides, most serious usability problems are related to the privacy heuristic.

Regarding the problems found for each user profile and heuristic (Figure 4), we have found that the heuristic with greater number of problems detected are the user profiles of general manager and caregiver manager. The exception happens in heuristic "privacy" where the user profile with more detected problems is the caregiver.

On the heuristic 4, consistency and standards, the profile with greater percentage of problems is the general manager ( $56 \%$ ), followed by the caregiver manager (28\%) and the caregiver (16\%), but there are not found problems with family member profile.

Heuristic 8, aesthetic and minimalist design, the profiles with higher percentage of problems are the general manager (46\%) and the caregiver manager (46\%), however the caregiver profile presents only $8 \%$ of the problems.

Related to the heuristic 11 , privacy, the profile with greater percentage of problems is the caregiver (45\%), followed by the caregiver manager (33\%) and the general manager $(22 \%)$.

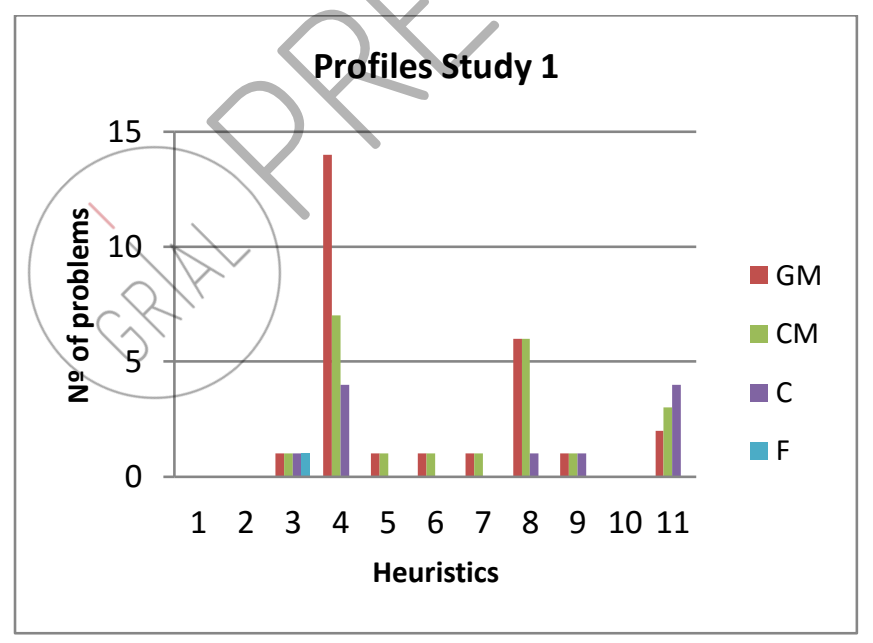

Figure 4. Number of problems found in study 1 of SocialNet clustered by user profile and heuristic

$\mathrm{GM}=$ General Manager; $\mathrm{CM}=$ Caregiver Manager $; \mathrm{C}=$ Caregiver $; \mathrm{F}=$ Family Member/Authorized

\subsubsection{Discussion}

The outcomes of the quantitative analysis of the data allow inferring that SocialNet is not consistency in terms of the names of the sections, buttons and content thereof. Problems that the expert related to the heuristic of "Consistency and standards", are detailed as: the existence of names of sections that do not match the page where we are, or the presence of buttons that lead nowhere. A homogenization of the names is necessary because situations like these generate the feeling of having made a mistake.

Problems for the heuristic of "Aesthetic and minimalist design" describe situations as: the presence of information that has no utility to the user, or checkboxes not aligned with the text to which is linked. The presence of strange information is a distraction that interferes with navigation. 
Related to heuristic "Privacy", the found problems make mention to: allowed access to profiles that are supposed to have no permission to access and profiles forbidden for performing certain steps and however have to access to them. This is a key point of the social networking information for generating confidence in users, and because of this should be protected as much as possible.

The criticality level of the problems encountered in these heuristics is medium-high stressing that the most serious problems are in the heuristic "privacy". Considering that this is a very important aspect, these problems should immediately correct.

In terms of application profiles has been observed that profiles in which more problems are found are those who has more tasks assigned as caregiver manager or the general manager, i.e. they have more permissions which makes you have to carry out more complex tasks and therefore the interface is more complex. When the permissions are less, the interface becomes simpler.

These data have been taken into account to carry out the redesign of SocialNet.

\subsubsection{Redesign based on the results of the evaluation of the study 1}

The analysis of the obtained results make necessary to carry out changes on the interface of SocialNet. The following problems were detected and the solutions adopted in the different heuristics:

Heuristic 3 (freedom and control of the user):

- Non-existence in the form of publication of the wall of the patient and so, the possibility for correcting errors easily. The solution implemented was a button for allowing "undo" or "cancel".

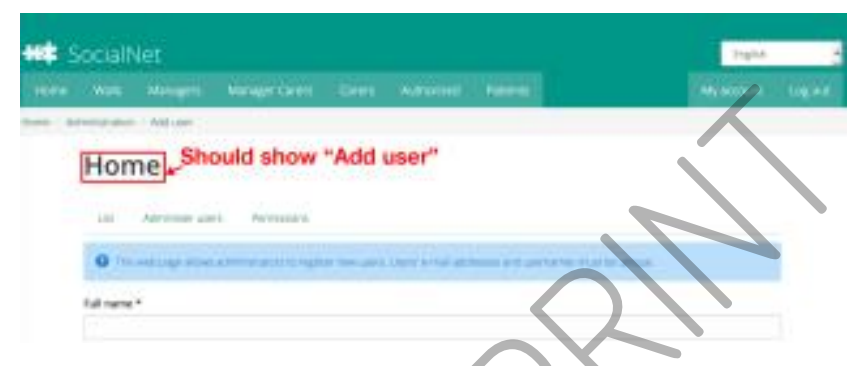

Figure 5. Page adding user prior to the redesign

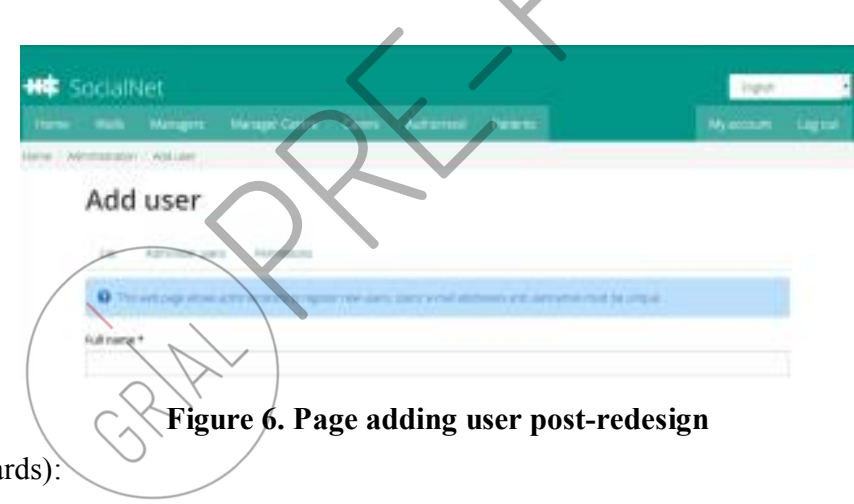

Heuristic 4 (consistency and standards):

- $\quad$ Existence of labels increasing confusion. A homogenization of the text had to be made. (Figure 5 and Figure 6)

- Add content link appears hidden in a drop-down menu as unique alternative. It was decided to show it as a fixed link.

- A button that leads to nowhere. It was decided to delete it.

- Duplicate links (which lead to the same site). It was decided to dispense with one of them.

Heuristic 5 (prevention of errors):

- Appearance of an error message that does not clarify the type of error to the user. Clarifying the error to the user amended it.

Heuristic 6 (better recognize that memorize):

- A button that leads to the other two options, one of which is the same one that indicates the button, but another one is difficult to relate. The solution was to develop two flaps for being watched easier by the user.

Heuristic 7 (flexibility and efficiency of use):

- The function "Edit" is very accessible in the list of users but other functions as lock, unlock or remove have very complicated its use, because it is necessary to go across the list for selecting an user and then go across the list again for selecting the function. In this case the solution was linked to make accessible all of these options, just close to every user.

Heuristic 8 (aesthetic and minimalist design): 
- $\quad$ Buttons and check boxes out of margin. They were properly looking for not to break the form design (Figure 7 and Figure 8).

- There were buttons appearing in user profiles with functions that do not correspond. In these cases, it was chosen to remove these buttons, as if these user profiles do not allow taking any action, his presence saturates the unnecessary information page.

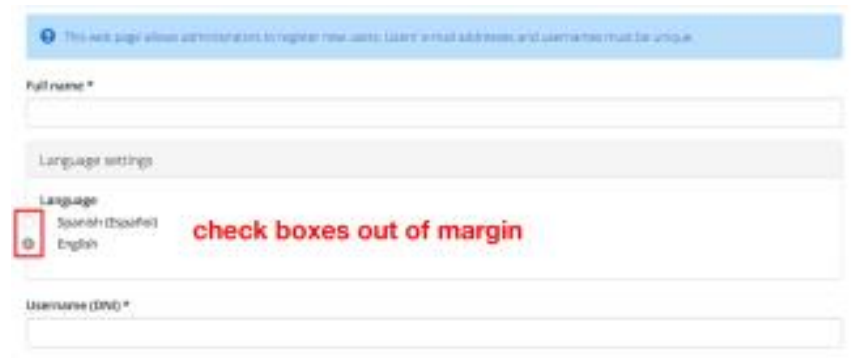

Figure 7. Form. Buttons language prior to the redesign

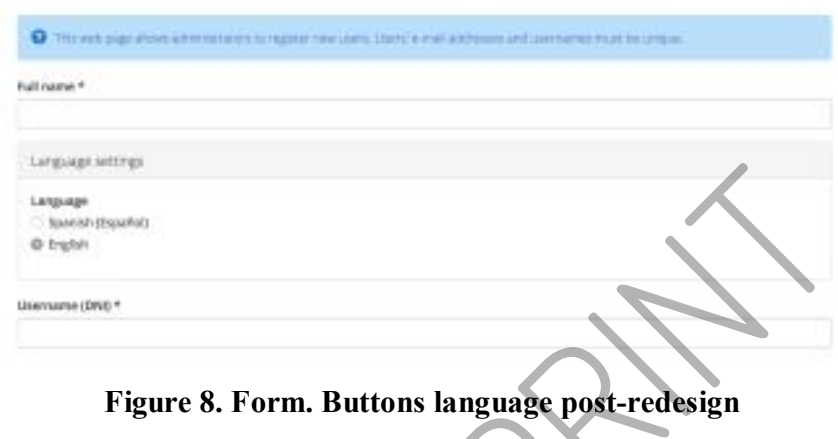

Heuristic 9 (helps the user recognize and recover from errors):

- In the registration form are fields without specifying the meaning of them to the user. The solution was to specify their meaning to the top of the page.

Heuristic 11 (Privacy):

- There were problems in terms of permissions and steps that correspond to the different roles. A review of it was carried out to adapt them to each user's profile.

\subsection{Study 2}

The second study is carried out after resolving the problems detected during the study described in the previous section. The profile selected to review the new version of the functional prototype of SocialNet is a user with experience using online tools and huge knowledge about SocialNet, specifically, the same expert that did the first review.

During this second phase of the heuristic evaluation the expert has followed the same steps of the first study in order to compare the differences between them.

\subsubsection{Results}

The results of this second evaluation are shown in a table that listed the number of problems clustered by heuristic and the number of problems clustered according to the user profile and heuristic. Figure 9 shows the identified problems clustered by heuristic. Only four of the eleven heuristics had problems, specifically in the heuristic 3, 4, 8 and 11, which correspond to freedom and control of user (1 problem), consistency and standards (5 problems), aesthetic design and minimalist (3 problems) and privacy (12 problems). No problem was found in the other of heuristics. 


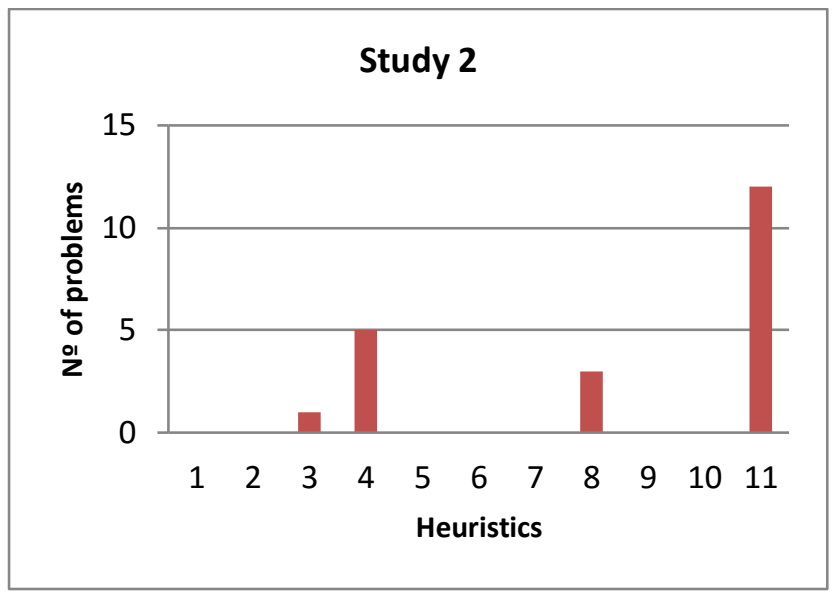

Figure 9. Number of problems found by heuristic in the study 2 of SocialNet

Regarding criticality level of the problems found in this second evaluation, most of the heuristics have medium-low level problems, except privacy where most of the problems are high criticality level. The results can be seen in the chart below (Figure 10).

In terms of problems found by user profile and heuristic, the profiles with most detected problems are general manager and caregiver manager. The exception, as it happened in the first study, arises in heuristic 11, because in this case, the user profile with more identified problems is the caregiver manager.

In heuristic 3, freedom and user control, is found a problem that appears in all profiles.

On the heuristic 4, consistency and standards, the profiles with higher percentage of problems are general manager (45\%) and caregiver manager (45\%), but caregiver profile only presents $10 \%$ problems.

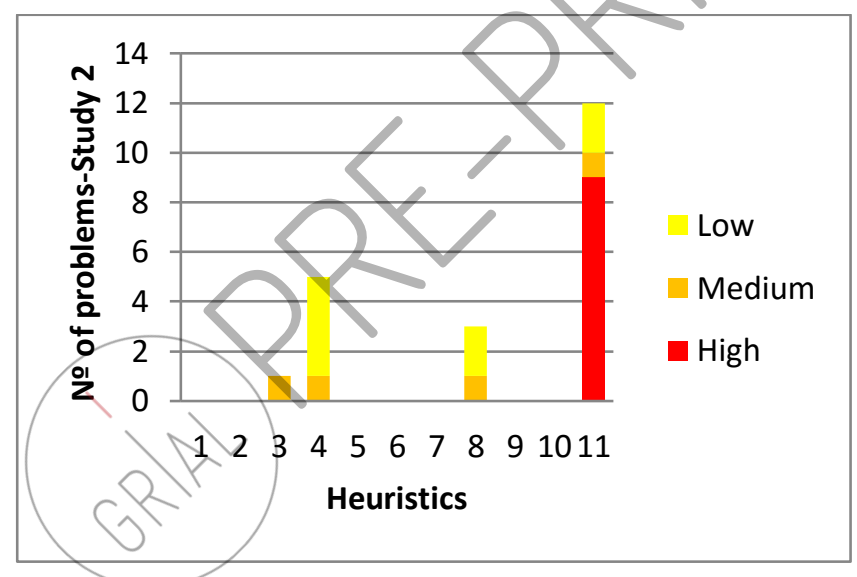

Figure 10. Criticality Level of the problems found in study 2

Related to Heuristic 8, aesthetic and minimalist design, the profiles with problems are general manager (50\%) and caregiver manager $(50 \%)$.

Finally, on the heuristic 11, privacy, the profile with greater percentage of problems is the caregiver manager ( $72 \%$ ), followed by general manager (18\%) and caregiver (10\%). 


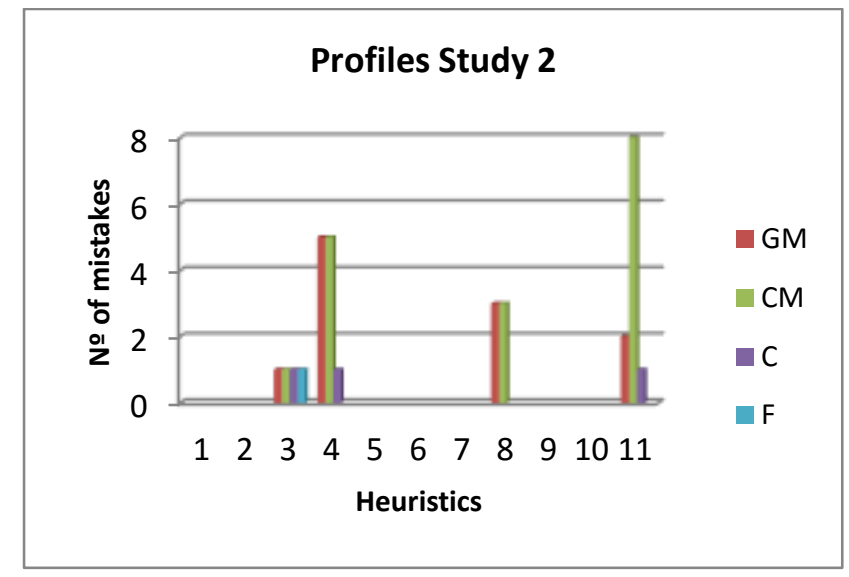

Figure 11. Number of problems by user profile and heuristic in SocialNet study 2

$\mathrm{GM}=$ General Manager; $\mathrm{CM}=$ Caregiver Manager ; $\mathrm{C}=$ Caregiver ; $\mathrm{F}=$ Family Member/Authorized

\subsubsection{Discussion}

The result of the analysis of data from this second study reveals, as expected, a substantial reduction of problems compared to the first study (Figure 12). Indeed, problems have only been found in four of the eleven heuristics. For instance, the problems found by the expert related to the heuristic 4 "consistency and standards", were reduced in a 70\%, and so the found problems were minimal being mainly related to the names given to certain buttons, headings or tab, which could improve the user experience.

On the heuristic of "Aesthetic and minimalist design" the problems were reduced in a $60 \%$. Most of them were referred to a simpler design and usable for some elements of the interface, which should be good for the navigation.

Related to the heuristic "Privacy" found problems were increased by $25 \%$ with respect to those found in the first study. This increment occurred especially in the profile "caregiver manager" which, could be due to the greater knowledge of evaluator about the SocialNet after the first study. The found problems were linked to the permissions for different user profiles. So, after correction of these mistakes, the level of protection available to the data that a user enters in the network has been increased. It is necessary take into account the key point of the social networking information for generating confidence in users.

Regarding criticality level of the problems found in the most of the heuristics is medium-low, but the most serious problems are in the heuristic "privacy". Because this is a very important aspect, these problems should immediately correct.

In terms of usage profiles, as it happened in the first study, the profiles with more problems are linked to more tasks assigned as caregiver manager or general manager.

Although the mistakes have been redueed substantially, after this second study continue appearing problems. The goal of the evaluator to perform various studies of the same application is to detect problems that at first step were not found, perhaps because in a first approach to SocialNet was difficult to take in consideration. Besides, it is necessary to underline the difficulties for evaluating the privacy of information or the hierarchy of permissions. Because of this, in the first stage the assessment was performed at a superficial level because the evaluator hadn't enough knowledge about the software. However, when more experience in the system is being reached, the evaluation can be made with more accuracy. So, perhaps best results can be obtained with a single evaluator who re-evaluates the application instead of several evaluators who assess it only once.

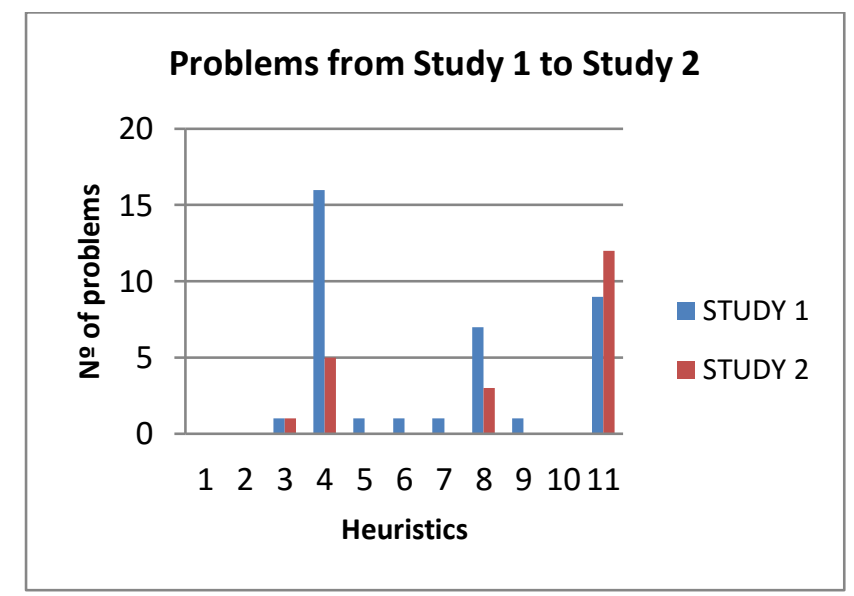

Figure 12. Comparison of the number of problems found by heuristic in studies 1 and 2 of SocialNet 


\subsubsection{Redesign based on the results of the evaluation of the study 2}

After the analysis of the obtained results, changes were carried out on the interface of SocialNet. The following are the problems and the solutions adopted in each case relating to the different heuristics:

Heuristic 3 (freedom and control of the user):

- Non-existence in the form of publication of the possibility of rectifying errors. This problem was already found in the first study, but was not resolved at that time. It was decided to implement a button "Cancel" (Figure 13).

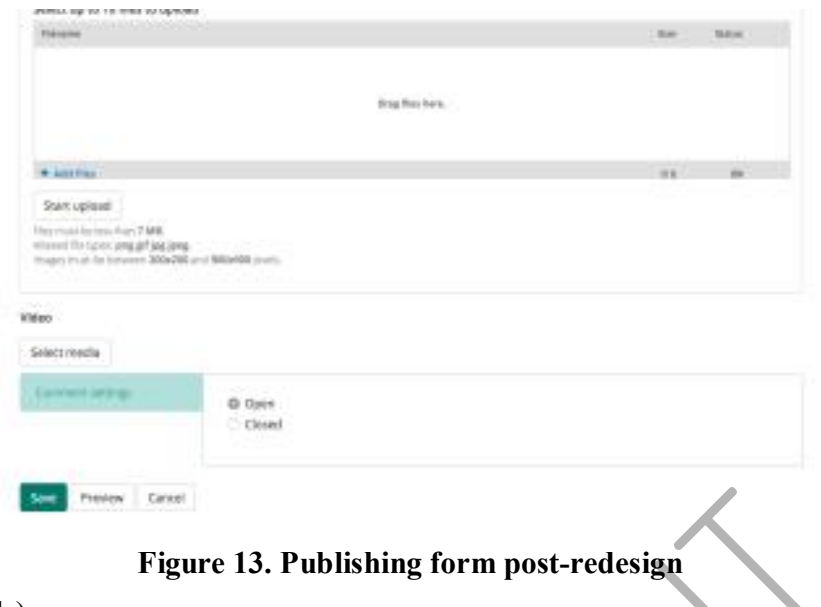

Heuristic 4 (consistency and standards):

- Existences of tags whose text may be confuse. A correction of the text is carried out.

Heuristic 8 (aesthetic and minimalist design):

- Some features of the User Manager show a tangled appearance. Reorganization was proposed, so that their appearance improved visually.

Heuristic 11 (Privacy):

- Emerged problems regarding permissions and steps that correspond to the different roles. All of it was reviewed to adapt them to each user profile again.

\section{CONCLUSIONS}

The goal of this study has been evaluating the usability of SocialNet. The intention from the outset has been that all users may have an effective, efficient interaction and a satisfactory user experience.

In this paper, we have described an assessment of the usability through heuristic evaluation based on a double study carried out by a single evaluator. The results suggest this methodołogy is very useful, practical, low cost and easy to implement. This technique has provided valuable data about the usability problems of SocialNet. Highlight the identified issues related to consistency, design and privacy. Those issues have been redesigned and have been included in the tool as part of the iterative development process.

We also have found that the second usability evaluation performed by the same evaluator has revealed new findings, which might suggest that there could be a relationship between previous experience with the social network and a better capacity of the evaluator to find aspects to improve in the application.

We should compare the reliability of the double assessment carried out by one evaluator with the one carried out by more than one evaluators, analyzing pros and cons.

Finally, the use of the heuristic evaluation should be complemented with a user test that is being carried out and it will make possible to observe the real problems of users and evaluate their experience using SocialNet.

\section{ACKNOWLEDGEMENTS}

The authors wish to thank the INTRAS Foundation (http://www.intras.es) and the Research Group in InterAction and eLearning (GRIAL) of the University of Salamanca http://grial.usal.es for their contributions and support.

This research work has been carried out within the University of Salamanca PhD Programme on Education in the Knowledge Society scope (http://knowledgesociety.usal.es) and was supported by the Spanish Ministerio de Educación, Cultura y Deporte under a FPU fellowship (FPU014/04783).

\section{REFERENCES}

[1] Fernandez, A., Insfran, E., and Abrahão, S., 2011. Usability evaluation methods for the web: A systematic mapping study. Information and Software Technology 53, 8, 789-817. 
[2] García-Holgado, A., Cruz-Benito, J., and García-Peñalvo, F.J., 2015. Analysis of Knowledge Management Experiences in Spanish Public Administration. In Proceedings of the Third International Conference on Technological Ecosystems for Enhancing Multiculturality (TEEM'15) (Porto, Portugal, October 7-9, 2015) ACM, New York, NY, USA, 189-193. DOI= http://dx.doi.org/10.1145/2808580.2808609.

[3] García-Holgado, A. and García-Peñalvo, F.J., 2014. Knowledge management ecosystem based on drupal platform for promoting the collaboration between public administrations. In Proceedings of the Second International Conference on Technological Ecosystems for Enhancing Multiculturality (TEEM'14) (Salamanca, Spain, October 1-3, 2014), F.J. García-Peñalvo Ed. ACM, New York, NY, USA, 619-624. DOI= http://dx.doi.org/10.1145/2669711.2669964.

[4] García-Holgado, A., García-Peñalvo, F.J., Hernández-García, Á., and Llorens-Largo, F., 2015. Analysis and Improvement of Knowledge Management Processes in Organizations Using the Business Process Model Notation. In New Information and Communication Technologies for Knowledge Management in Organizations: 5th Global Innovation and Knowledge Academy Conference, GIKA 2015, Valencia, Spain, July 14-16, 2015, Proceedings, D. Palacios-Marqués, D.R. Soriano and K.H. Huarng Eds. Springer International Publishing, 93-101.

[5] García-Peñalvo, F.J., 2016. Presentation of the GRIAL research group and its main research lines and projects on March 2016.

[6] García-Peñalvo, F.J. and Conde, M.Á., 2014. Using informal learning for business decision making and knowledge management. Journal of Business Research 67, 5, 686-691. DOI= http://dx.doi.org/10.1016/j.jbusres.2013.11.028.

[7] García-Peñalvo, F.J., Franco Martín, M., García-Holgado, A., Toribio Guzmán, J.M., Largo Antón, J., and Sánchez-Gómez, M.C., 2016. Psychiatric patients tracking through a private social network for relatives: development and pilot study. Journal of Medical Systems 40, 7, Paper 172. DOI= http://dx.doi.org/10.1007/s10916-016-0530-5.

[8] García-Peñalvo, F.J., Rodríguez Conde, M.J., Seoane, A.M., Conde, M.Á., Zangrando, V., and García-Holgado, A., 2012. GRIAL (GRupo de investigación en InterAcción y eLearning), USAL. In Revista Iberoamericana de Informática Educativa ADIE, 85-94.

[9] Girón, M., Nova-Fernández, F., Mañá-Alvarenga, S., Nolasco, A., Molina-Habas, A., Fernández-Yañez, A., Tabarés-Seisdedos, R., and Gómez-Beneyto, M., 2015. How does family intervention improve the outcome of people with schizophrenia? Social psychiatry and psychiatric epidemiology 50, 3, 379-387. DOI= http://dx.doi.org/10.1007/s00127-014-0942-9.

[10] Gulati, A. and Dubey, S.K., 2012. Critical analysis on usability evaluation techniques. International Journal of Engineering Science and Technology 4,3 .

[11] Hermawati, S. and Lawson, G., 2016. Establishing usability heuristics for heuristics evaluation in a specific domain: Is there a consensus? Applied Ergonomics 56, 34-51.

[12] Hollingsed, T. and Novick, D.G., 2007. Usability inspection methods after 15 years of research and practice. In Proceedings of the 25th annual ACM international conference on Design of communication ACM, 249-255.

[13] Hwang, W. and Salvendy, G., 2010. Number of people required for usability evaluation: the 10\&plusmn;2 rule. Commun. ACM 53, 5, 130-133. DOI= http://dx.doi.org/10.1145/1735223.1735255.

[14] Jeavons, B.J. and Knaddison, G.J., 2014. Drupal Security White Paper.

[15] Kemp, S., 2016. Digital in 2016. We Are Social, http://www.slideshare.net/wearesocialsg/digital-in-2016.

[16] Mening, R., 2014. WordPress vs Joomla vs Drupal + CMS "comparison chart".

[17] Naslund, J., Aschbrenner, K., Marsch, L., and Bartels, S., 2016. The future of mental health care: peer-to-peer support and social media. Epidemiology and psychiatric sciences 25, 2, 113-122. DOI= http://dx.doi.org/10.1017/S2045796015001067.

[18] Nielsen, J., 1994. Heuristic evaluation. In Usability inspection methods, J. Nielsen and R.L. Mack Eds. John Wiley \& Sons, Inc., 25-62.

[19] Nielsen, J., 2016. How to Conduct a Heuristic Evaluation.

[20] Nielsen, J. and Molich, R., 1990. Heuristic evaluation of user interfaces. In Proceedings of the SIGCHI conference on Human factors in computing systems ACM, 249-256.

[21] Pierotti, D., 1995. Heuristic evaluation-a system checklist. Xerox Corporation.

[22] Rackspace, 2013. CMS Comparison: Drupal, Joomla and Wordpress.

[23] Wilson, C., 2013. User interface inspection methods: a user-centered design method. Newnes.

[24] Zazelenchuk, D., 2006. Heuristic Evaluation and its alternatives, http://www.userfocus.co.uk/articles/heuristics.html. 